\title{
VERSIONS OF KOEBE 1/4 THEOREM FOR ANALYTIC AND QUASIREGULAR HARMONIC FUNCTIONS AND APPLICATIONS
}

\author{
Miodrag Mateljević
}

\begin{abstract}
In this paper we mainly survey results obtained in [MM3]. For example, we give an elementary proof of two versions of Koebe $1 / 4$ theorem for analytic functions (see Theorem 1.2 and Theorem 1.4 below). We also show a version of the Koebe theorem for quasiregular harmonic functions. As an application, we show that holomorphic functions (more generally quasiregular harmonic functions) and their modulus have similar behavior in a certain sense.
\end{abstract}

\section{Two versions of Koebe $1 / 4$ theorem for analytic functions}

This paper can be considered as the review of some results presented in [MM3], but it also contains new results and proofs. We will use the following notation. If $r>0$ and $a$ is a complex number $B(a ; r)=\{z \in \mathbb{C}:|z-a|<r\}$ is the open circular disc with center at $a$ and radius $r$. Also we use notation $\Delta_{r}=B(0, r)$ and $\Delta=\Delta_{1}$. First, we introduce a particulary interesting class of conformal mappings of the disc, the class $S$. We denote by $S$ the class of holomorphic functions $f$ in $\Delta$ which are injective and satisfy the normalization conditions $f(0)=0$ and $f^{\prime}(0)=1$.

The proof of Koebe's One-Quarter Theorem is based on the extremal property of the Grötzsch annulus, which we first need to discuss.

1.1. Grötzsch Theorem and Koebe's One-Quarter Theorem. If $\Omega$ is a double connected domain, by $M(\Omega)$ we denote the modulus of $\Omega$; and for a given family of curves $\Gamma$ by $M(\Gamma)$ modulus of the family of curves $\Gamma$. If $\Omega$ is a double connected domain and $E_{1}$ and $E_{2}$ the components of $\partial \Omega$ the extremal distance $d_{\Omega}\left(E_{1}, E_{2}\right)$ between $E_{1}$ and $E_{2}$ is the modulus of $\Omega$. Note that if $\Gamma$ is the family of curves which joins the components $E_{1}$ and $E_{2}$, then $d_{\Omega}\left(E_{1}, E_{2}\right)=1 / M(\Gamma)$ and if $\Gamma^{\prime}$ is the family of curves which separates the components $E_{1}$ and $E_{2}$ then $M(\Omega)=M\left(\Gamma^{\prime}\right)$.

Let $0<r<1$ and $c$ be any continum that contains $\{0, r\}$; and let $\Gamma, \Gamma_{0}$ and $\Gamma_{c}$ be the families of closed curves in the unit disk that separate $\{0, r\}, s=[0, r]$

2000 Mathematics Subject Classification: Primary 30C62, 30C80, 30C55; Secondary $30 \mathrm{H05.}$ 
and $c$ from the circumference respectively. There is an extremal metric $\rho_{0}$ for the family $\Gamma_{0}$, which is obviously symmetric with respect to $s$.

Given any $\gamma \in \Gamma$ we obtain a path $\gamma_{0}$ of equal $\rho_{0}$-length by reflecting part of $\gamma$ across $s$. Hence, we conclude $M(\Gamma)=M\left(\Gamma_{0}\right)$. Note the crucial role of symmetry.

Since $\Gamma_{c}$ is the smaller family than $\Gamma$, we find $M\left(\Gamma_{c}\right) \leqslant M(\Gamma)=M\left(\Gamma_{0}\right)$.

In view of conformal invariance, we get:

THEOREM 1.1 (Grötzsch Theorem). Of all the continua that join the point $R>1$ to $\infty$ the segment $[R,+\infty]$ has the greatest extremal distance from the unit circle.

It is convenient to introduce the notation $\mathbb{C}^{R}, R \geqslant 0$, for the complex plane cut along $[R,+\infty)$ and $\mathbb{C}_{r}^{R}=\mathbb{C}^{R} \backslash \overline{\Delta_{r}}$ for the complement of the closed disk $\overline{\Delta_{r}}$ and $[R,+\infty)$ (known as the Grötzsch annulus).

Theorem K (Koebe's One-Quarter Theorem). If $f \in S$ then $f(\Delta) \supset \Delta_{1 / 4}$.

Proof. Let $f \in \mathcal{S}, 0<\varepsilon<1, A_{\varepsilon}=\Delta \backslash \overline{\Delta_{\varepsilon}}, G_{\varepsilon}=f\left(A_{\varepsilon}\right)$, and $\delta=\delta_{f}=$ dist $(0, \partial f(\Delta))$. Since the modulus is a conformal invariant, it follows $M\left(G_{\varepsilon}\right)=$ $\frac{1}{2 \pi} \ln \frac{1}{\varepsilon}$.

For $0<\varepsilon<1$, let $\varepsilon_{*}=\min \{|f(z)|:|z|=\varepsilon\}$. Then

$$
M\left(C_{\varepsilon}^{\delta}\right)=\frac{1}{2 \pi} \ln \frac{4 \delta}{\varepsilon+o(\varepsilon)} .
$$

By the monotonous principle for modulus and Grötzsch's theorem, $M\left(G_{\varepsilon}\right) \leqslant$ $M\left(C_{\varepsilon_{*}}^{\delta}\right)$ and hence, since $\varepsilon_{*}=\varepsilon+0(\varepsilon)$,

$$
\ln \frac{1}{\varepsilon} \leqslant \ln \frac{4 \delta}{\varepsilon+0(\varepsilon)} .
$$

Now passing to the limit when $\varepsilon \rightarrow 0$, it follows $\frac{1}{4} \leqslant \delta_{f}$; that is $f(\Delta) \supset \Delta_{1 / 4}$.

1.2. Koebe $1 / 4$ theorem for analytic functions. EXAMPLE 1 . The example $f_{n}(z)=\frac{1}{n}\left(e^{n z}-1\right)$ shows that if $f$ is an analytic function on the unit disc $\Delta, f(0)=0$ and $\left|f^{\prime}(0)\right| \geqslant 1$, then there is no absolute constant $s$ such that the disc $B(0, s)$ belongs to $f(U)$.

This example shows the hypothesis that $f \in S$ that is, roughly speaking, injectivity of $f$ is essential for validity of Koebe's One-Quarter Theorem. It is a natural question: whether exists an appropriate generalization of this theorem to functions which are not injective.

After writing several versions of this paper we have found very simple proof of the following result which seems to be an appropriate generalization of Koebe one-quarter theorem (with the best constant $\frac{1}{4}$ ). In order to state the theorem we need to introduce some notations.

Recall, by $\Delta$ we denote the unit disc in the complex plane. If $z$ and $w$ are complex numbers, by $\Lambda(z, w)$ we denote the half-line $\Lambda(z, w)=\{z+\rho(w-z): \rho \geqslant 0\}$ and we write $\Lambda(w)=\Lambda(0, w)$. If $\theta \in \mathbb{R}$ we also write $\Lambda_{\theta}$ and $\Lambda_{a}^{\theta}$ respectively instead of $\Lambda\left(0, e^{i \theta}\right)=\left\{\rho e^{i \theta}: \rho \geqslant 0\right\}$ and $\Lambda\left(a, a+e^{i \theta}\right)$. 
By $\omega=\omega_{f}$ we denote the modulus of continuity of $f$.

LEMMA 1.1 (Koebe lemma for analytic functions). Suppose that $f$ is an analytic function on the closed unit disc $\bar{\Delta}, f(0)=0$ and $\left|f^{\prime}(0)\right| \geqslant 1$. Then for every $\theta \in \mathbb{R}$ there exists a point $w$ on the half-line $\Lambda_{\theta}$ which belongs to $f(\bar{\Delta})$, such that $|w| \geqslant \frac{1}{4}$.

Proof. Contrary, suppose there exists $\theta$ for each the theorem is not true. Without loss of generality we can assume that

$$
f(\bar{\Delta}) \text { does not intersect } \Lambda=[1 / 4, \infty) .
$$

Then Koebe function $k_{0}$, which is defined by $k_{0}(z)=\frac{z}{(1+z)^{2}}$, maps $\Delta$ onto $\mathbb{C} \backslash \Lambda$ and by the subordination principle $\left|f^{\prime}(0)\right| \leqslant 1$. If $\left|f^{\prime}(0)\right|=1$, then $f=k_{0}$ and $f(1)=k_{0}(1)=1 / 4$, which is a contradiction with the hypothesis $\left(^{*}\right)$. Hence $\left|f^{\prime}(0)\right|<1$, which is again a contradiction with the hypothesis $\left(^{*}\right)$.

As an application of Lemma 1.1, we immediately obtain the following result, which we call the first version of Koebe theorem for analytic functions.

TheOREM 1.2 (first version of Koebe theorem for analytic functions). Let $B=$ $B(a ; r)$, $f$ holomorphic function on $\bar{B}, D=f(B)$, and $f(a)=b$. Then for every $\theta \in \mathbb{R}$ and $b \in D$, there exists a point $w$ on the half-line $\Lambda_{b}^{\theta}$, which belongs to $f(\bar{B})$, such that $|w-b| \geqslant \rho_{f}(a)$, where $\rho_{f}(a):=\frac{1}{4} r\left|f^{\prime}(a)\right|$. In particular, there exists a point $\omega \in D$ such that $|\omega|-|b|=|\omega-b| \geqslant \rho_{f}(a)$.

Proof. If $f^{\prime}(a) \neq 0$, applying Lemma 1.1 to the function $s(f(a+r z)-b)$, where $s=1 / r f^{\prime}(a)$, immediately gives the result.

In connection with the first version of Koebe theorem for analytic functions, Bloch theorem comes in mind. Namely, Bloch theorem states:

THEOREM 1.3 (Bloch theorem). If $f$ is a normalized holomorphic function on $\Delta$, that is $\left|f^{\prime}(0)\right| \geqslant 1$, then $f(\Delta)$ contains a disc of radius greater then an absolute constant.

This gives a version of the key estimate (2.2) with an absolute constant.

Suppose that the complement of $D$ is not empty; and let $d=d(w)=\operatorname{dist}(w, \partial D)$. Bloch theorem states there is an absolute constant $c$ and $w_{0} \in D$ such that the disk $B=B\left(w_{0} ; R\right)$ of radius $R=c \rho_{f}(a)$ belongs to $D$. It is clear that there is a point $w_{1}$ in the intersection of $D$ with the line $\Lambda$ which contains points 0 and $w_{0}$ such that $\left|w_{1}\right|-\left|w_{0}\right|=\left|w_{1}-w_{0}\right| \geqslant R$. Hence, $R \leqslant \omega_{|f|}(r)$. Thus, under conditions of Theorem 1.2, we have

$$
c r\left|f^{\prime}(a)\right| \leqslant \omega_{|f|}(r) .
$$

This estimate is crucial for applications in Section 2. Here, by $\omega=\omega_{f}$ we denote the modulus of continuity of $f$.

Using Koebe's One-Quarter Theorem for univalent function we can generalize Theorem 1.2, the first version of Koebe theorem for analytic functions. 
THEOREM 1.4 (second version of Koebe theorem for analytic functions). Let $B=B(a ; r)$, $f$ holomorphic function on $B, D=f(B), f(a)=b$ and let the unbounded component $D_{\infty}$ of $D$ is not empty; and let $d_{\infty}=d_{\infty}(b)=\operatorname{dist}\left(b, D_{\infty}\right)$. Then: (1) $r\left|f^{\prime}(a)\right| \leqslant 4 d_{\infty} ; \quad$ (2) If, in addition, $D$ is simply connected, then $D$ contains the disk $B(b, \rho)$ of radius $\rho$, where $\rho=\rho_{f}(a)=r\left|f^{\prime}(a)\right| / 4$.

ProOF. Let $D_{0}$ is the complement of $\overline{D_{\infty}}$ and $\varphi$ conformal mapping of $D_{0}$ onto $\Delta$ such that $\varphi(b)=0$ and let $\psi=\varphi^{-1}$; and $F=\varphi \circ f$. Then, by Koebe theorem for univalent function, the disk $B_{*}=B\left(b, R_{0}\right)$, where $R_{0}=\mid \psi^{\prime}\left(0 \mid / 4\right.$, belongs to $D_{0}$ and hence $R_{0} \leqslant d=\operatorname{dist}\left(b, \partial D_{0}\right)$, that is

$$
\mid \psi^{\prime}(0 \mid \leqslant 4 d
$$

By the Schwarz lemma,

$$
r\left|F^{\prime}(a)\right| \leqslant 1 \text {. }
$$

Since $\varphi^{\prime}(b) \psi^{\prime}(0)=1$, then $F^{\prime}(a)=\varphi^{\prime}(b) f^{\prime}(a)=f^{\prime}(a) / \psi^{\prime}(0)$, and hence it follows, by (1.3), that $r\left|f^{\prime}(a)\right| \leqslant\left|\psi^{\prime}(0)\right|$; and therefore since $d=d_{\infty}$, by (1.2) $r\left|f^{\prime}(a)\right| \leqslant 4 d_{\infty}$, that proves the part (1).

If $D$ is simply connected, then $d_{\infty}=d_{\infty}(b)=\operatorname{dist}\left(b, D_{\infty}\right)=d=\operatorname{dist}(b, \partial D)$, and this proves the part (2).

Suppose, in addition, that $f$ is holomorphic on $\bar{B}$. Then for every $\theta \in \mathbb{R}$ and $b \in D$, there exists a point $w$ on the half-line $\Lambda_{b}^{\theta}$, which belongs to $f(\bar{B})$, such that $|w-b| \geqslant \frac{1}{4} r\left|f^{\prime}(a)\right|$.

Thus, Theorem 1.2 is a special case of Theorem 1.4.

Note that, in general, $D=f(B)$ is not a simply-connected domain and the disk $B_{*}=B(b, R)$ does not belong to $D$ (see Example 1 and the next example).

ExAmple 2. Let $Q=Q_{r}=(-r, r)^{2}, r>2 \sqrt{2} \pi$, and $\phi(z)=\exp (z)$. Then $\phi(0)=\phi^{\prime}(0)=1$ and $\phi(Q)=A$, where $A=A_{r}=\{w: \exp (-r)<|w|<\exp (r)\}$ is a doubly-connected ring. By Theorem 1.4 [the second version of Koebe theorem for analytic function], $r\left|\phi^{\prime}(0)\right|=r \leqslant 4 d$, where $d=d_{\infty}(1)$. Since $Q_{r / \sqrt{2}} \subset \Delta_{r}$, it is easy to check that here, actually, $\exp (r / \sqrt{2})-1 \leqslant d_{\infty}(1)$. Hence, $d_{\infty}(1) \rightarrow+\infty$ when $r \rightarrow+\infty$. However, the disk $B(1 ; 1)$ does not belong to $A=A_{r}$ for any $r>0$.

\section{Applications}

As an application we prove Dyakonov's theorem. In discussion which follows, we suppose that $\alpha \in(0,1)$. By $\omega=\omega_{f}$ we denote the modulus of continuity of $f$.

Roughly speaking, the first and second version of Koebe theorem for analytic functions state that holomorphic functions have the same dilatation in all directions and it indicates similar behavior of holomorphic function and its modulus in certain sense and leads, via crucial estimate (2.3), to what we call geometric visual proof of Dyakonov's theorem[Dyk1] stated here as:

THEOREM A. If $f$ is an analytic function on $\Delta$, then $f$ belongs $\operatorname{Lip} \alpha$ if and only if $|f|$ belongs $\operatorname{Lip} \alpha$. 
The original proof of Theorem A was complicated; for simple proofs see $[\mathbf{P}]$, [MM1], and [MM3].

Namely, if $f$ belongs Lip $\alpha$, then, by triangle inequality, $|f|$ belongs Lip $\alpha$. Proof of the opposite result is more delicate as the next example indicates:

EXAMPLE 3. Let $R=R_{r}=(-r, r) \times(-\infty,+\infty)=\{z:-r<x<r\}, r>0$, $\varphi$ conformal mapping of $\Delta$ onto $R$ such that $\varphi(0)=0$ and $\phi(z)=\phi_{n}(z)=$ $\exp (\varphi(z) / n)$. Then $\omega_{\phi_{n}}(1) \geqslant 2$ and $\phi_{n}(\Delta)=A$, where $A=A_{n}=\{w: \exp (-r / n)<$ $|w|<\exp (r / n)\}$, and therefore $\omega_{\left|\phi_{n}\right|}(1)=2 r / n+o(1 / n) \rightarrow 0$, when $n \rightarrow \infty$. Note that, by the triangle inequality, $\omega_{|f|} \leqslant \omega_{f}$. Since $\phi_{n}, n \geqslant 1$, are not continuous on $\bar{\Delta}$, these function do not belong to $\bigcup_{0<\alpha<1} \operatorname{Lip}(\alpha)$ on $\Delta$.

Even, if we consider the family $\mathcal{H}^{\infty}$ of bounded analytic function on the unit disk as a frame of investigation, there is no an absolute constant $K$ such that $\omega_{f} \leqslant K \omega_{|f|}$ for every $f \in \mathcal{H}^{\infty}$.

The following lemma which gives the estimate of modulus of derivative is an immediate corollary of the key estimate (2.3). It with the gradient growth lemma (see the appendix) yields immediately a proof that if $|f|$ belongs Lip $\alpha$, then $f$ belongs $\operatorname{Lip} \alpha$.

Lemma 2.1. Let $f$ be an analytic function on $\Delta$. Then

$$
(1-|z|)\left|f^{\prime}(z)\right| \leqslant 4 \omega(1-|z|), \quad z \in \Delta,
$$

where $\omega=\omega_{|f|}$ is the modulus of continuity of $|f|$. In addition, if $|f|$ belongs $\operatorname{Lip} \alpha$, then

$$
(1-|z|)\left|f^{\prime}(z)\right| \leqslant 4(1-|z|)^{\alpha}, \quad z \in \Delta .
$$

The estimate (4.2) in the appendix shows that the constant 4 can be replaced by 2 in the above inequalities.

Notes: In the appendix, we prove a version of Schwarz lemma (the estimate (4.1)), which is very close to classical one (and probably known to the experts in the subject), and we derive the estimate (4.2) as an immediate corollary of it.

Proof. Let $z \in \Delta, r=s(1-|z|), 0<s<1, B=B(z ; r), w=f(z)$ and $\tilde{B}=f(B)$. By Theorem 1.2 [the first version of Koebe theorem for analytic function], there is a point $w_{1}$, which belongs to $\tilde{B} \cap \Lambda(w)$, such that

$$
\left|w_{1}-w\right| \geqslant \frac{1}{4}\left|f^{\prime}(z)\right| r
$$

Let $z_{1}$ be preimage of $w_{1}$. Since

$$
\left|w_{1}-w\right|=|| w_{1}|-| w||=|| f\left(z_{1}\right)|-| f(z)|| \text { and }\left|z_{1}-z\right| \leqslant r,
$$

then

$$
s(1-|z|)\left|f^{\prime}(z)\right| \leqslant 4 \omega[s(1-|z|)], \quad z \in \Delta .
$$

Hence, if $s \rightarrow 1_{-}$, then (2.1) follows from (2.3).

Note that if $|f|$ belongs Lip $\alpha$, then (2.1) reduces to (2.2). 
Using known result (see for example $[\mathbf{R}]$, Lemma 6.4.8) and Lemma 2.1 we get Dyakonov's theorem: If $f$ is an analytic function on $\Delta$, then $f$ belongs Lip $\alpha$ if and only if $|f|$ belongs $\operatorname{Lip} \alpha$. Namely, if $f$ belongs $\operatorname{Lip} \alpha$, then, by triangle inequality, $|f|$ belongs $\operatorname{Lip} \alpha$. If $|f|$ belongs $\operatorname{Lip} \alpha$, then it follows from the inequality (2.2) and Lemma 6.4.8 [R] (stated as Lema 4.1 [the gradient growth lemma] in the appendix), that $f$ belongs $\operatorname{Lip} \alpha$.

Having in mind the previous proof of Theorem A, it is clear that it can be based on the estimate (4.2), which easily follows from Schwarz lemma.

We can also get Theorem A as an immediate corollary of Bloch theorem. Namely, Bloch theorem states if $f$ is a normalized holomorphic function on $\Delta$, that is $\left|f^{\prime}(0)\right| \geqslant 1$, then $f(\Delta)$ contains a disc of radius greater then an absolute constant. This gives a version of the key estimate (2.2) with an absolute constant.

Try to understand (investigate) which property of holomorphic function is essential for similar behavior of holomorphic function and its modulus in certain sense, leads to generalizations of Theorem A.

In [MM1, MM2], we have outlined a proof of a version of Theorem 1.2 (and therefore Theorem A for quasiregular harmonic mappings and have indicated that it is true for classes of functions which include pseudo-holomorphic functions, real harmonic functions of several variables), holomorphic functions of several variables, etc (see also [MM1, MM2] and [Dyk2] for further comments and references).

By $B^{n}=\left\{x \in R^{n}:|x|<1\right\}$ we denote the unit ball and by $\mathbb{S}^{n}$ the unit in $R^{n}$.

For example, we can prove the following:

Proposition 2.1. Suppose that $0<\alpha<1, h: \mathbb{B}^{n} \rightarrow \mathbb{R}^{m}$ is a Euclidean harmonic mapping and continuous on $\overline{\mathbb{B}}^{n}$. The following conditions are equivalent:

(a) $|h(x)-h(y)| \leqslant m|x-y|^{\alpha}, x, y \in \mathbb{S}^{n} ;$

(b) $h$ belongs to $\operatorname{Lip}(\alpha)$ on $\mathbb{B}^{n}$;

(c) $|\operatorname{grad} h(x)| \leqslant A(1-|x|)^{\alpha-1}, x \in \mathbb{B}^{n}$.

If, in addition, $h$ is real then the following conditions are equivalent

(d) $h$ belongs to $\operatorname{Lip}(\alpha)$ on $\mathbb{B}^{n}$;

(e) $|h|$ belongs to $\operatorname{Lip}(\alpha)$ on $\mathbb{B}^{n}$;

(f) $|h|$ belongs to $\operatorname{Lip}(\alpha)$ on $\mathbb{S}^{n}$.

If $h$ is real and $|h|$ belongs to $\operatorname{Lip}(\alpha)$, on $\mathbb{S}^{n}$, then $h$ belongs to $\operatorname{Lip}(\alpha)$ on $\mathbb{S}^{n}$.

We outline a simple proof. First we verify that $(f)$ is equivalent to

(g) $h$ belongs to $\operatorname{Lip}(\alpha)$ on $\mathbb{S}^{n}$.

Suppose that $(f)$ holds, that is $h$ belongs to $\operatorname{Lip}(\alpha)$ on $\mathbb{S}^{n}$ and let $x_{1}, x_{2} \in \mathbb{S}^{n}$ and $h\left(x_{1}\right) \leqslant 0 \leqslant h\left(x_{2}\right)$. Then there is $x_{0}$ on the spherical geodesic arc which joins $x_{1}$ and $x_{2}$ such that $h\left(x_{0}\right)=0$. Hence

$$
\left|h\left(x_{2}\right)-h\left(x_{1}\right)\right| \leqslant\left|h\left(x_{2}\right)-h\left(x_{0}\right)\right|+\left|h\left(x_{0}\right)-h\left(x_{1}\right)\right| \leqslant c\left|x_{2}-x_{0}\right|^{\alpha}+c\left|x_{1}-x_{0}\right|^{\alpha} .
$$

Suppose that, for example, $\left|x_{2}-x_{0}\right| \leqslant\left|x_{1}-x_{0}\right|$. Then

$$
\left|h\left(x_{2}\right)-h\left(x_{1}\right)\right| \leqslant 2 c\left|x_{1}-x_{0}\right|^{\alpha} \leqslant 2 c\left|x_{2}-x_{1}\right|^{\alpha} .
$$

Thus, we get $(\mathrm{g})$. It is known that $(\mathrm{g})$ implies $(\mathrm{d})$; hence the statement follows. 
It is clear that if $D \subset \mathbb{R}^{n}$ is convex domain, $0<\alpha \leqslant 1, f$ continuous real function on $D$, then $f$ belongs to $\operatorname{Lip}(\alpha)$ if and only if $|f|$ belongs to $\operatorname{Lip}(\alpha)$ on $D$.

After writing a version of the paper, D. Kaljaj informed the author about the paper $[\mathbf{P 1}]$, in which it is proved that (f) is equivalent to (d).

\section{Koebe theorem for quasiregular harmonic functions}

It is well known that if $f$ is a quasiconformal mapping defined on a region $G$, then the function $f_{z}$ is nonzero a.e. in $G$. The function $\mu_{f}=f_{\bar{z}} / f_{z}$ is therefore a well defined bounded measurable function on $G$, called the complex dilatation or Beltrami coefficient of $f$. Let $\mu$ be a complex dilatation on $\Delta$. For $z \in \Delta$, we define $\mu^{+}(z)=\operatorname{ess} \sup \{|\mu(\zeta)|:|\zeta|=|z|\}$, where ess sup is taken with respect to the arc length (the angular measure) on the circle of the radius $|z|$. Set

$$
\tau=\tau(f)=\tau_{f}=\int_{0}^{1} \frac{2 \mu^{+}(t)}{1+\mu^{+}(t)} \frac{d t}{t}, \quad \text { and } \quad \delta=\delta(f)=\delta_{f}=\operatorname{dist}(f(0), \partial f(\Delta)) .
$$

Note that $\mu^{+}$is a radial function.

Using the method of extremal length (more precisely the Grötzsch theorem) we proved [MM3]:

THEOREM 3.1. Suppose the above notation and

(a) $f$ is a quasiconformal mapping on $\Delta_{r}$ for all $0<r<1$, and

(b) $f(0)=0$ and $f$ is conformal at 0 .

Then

$$
\left|f^{\prime}(0)\right| \leqslant 4 \delta_{f} e^{\tau(f)} .
$$

Note that $\tau$ does not converges in general. If $\tau$ converges, by Theorem 6.1 in $[\mathbf{L V}] f$ is conformal at 0 .

If $f$ is a conformal mapping, then $\mu_{f}$ equals 0 on $\Delta$ and therefore $\tau(f)=0$. Hence, Koebe's one-quarter theorem follows from the estimate (3.1).

In this section, we will show that a version of Theorem 1.2 holds for quasiregular harmonic functions. For basic definitions and results we refer to $[\mathbf{A h 2}],[\mathbf{L V}])$ and [Ri] books. First, we need to introduce some notations and results.

Every harmonic function $f$ in $\Delta$ can be written in the form $f=\bar{g}+h$, where $g$ and $h$ are holomorphic functions in $\Delta$. For $f \in S_{H}^{0}$ (see [Cl-Sh] for the notation), $\left|g^{\prime}(z)\right| \leqslant|z|\left|h^{\prime}(z)\right|$.

LEMMA 3.1. Let $f$ be a diffeomrphism of $\Delta$,

(1) $|\bar{\partial} f(z)| \leqslant|z||\partial f(z)|$ for $z \in \Delta$, and

(2) $f(z)=z+O\left(|z|^{\beta}\right)$ for some $\beta>1$ as $z \rightarrow 0$.

Then $f(\Delta) \supset \Delta_{1 / 16}$.

Clunie and Sheil-Small proved the lemma for harmonic mappings $f=\bar{g}+h \in$ $S_{H}^{0}$. For the proof of lemma in general, one can repeat their approach using $\partial f(z)$ and $\bar{\partial} f(z)$ respectively instead of $h^{\prime}$ and $g^{\prime}$. The details are left to the interested reader. The lemma also appears in $[\mathbf{H e}-\mathbf{S c}],[\mathbf{H e}-\mathbf{P o}]$. The lemma is true if the hypothesis (2) is replaced by the hypothesis 
(3) $f$ is conformal at 0 and $f^{\prime}(0)=1$.

We now outline an argument which shows that the lemma is also an immediate corollary of Theorem 3.1. Let $\mu=\mu_{f}$. By the hypotheses of the lemma $|\mu(z)| \leqslant|z|$, $z \in \Delta$, and we obtain first $\mu^{+}(t) \leqslant t, 0 \leqslant t<1$, and therefore $\tau \leqslant 2 \ln 2$. Hence, since the hypotheses (2) implies the hypotheses (3), an application of Theorem 3.1 gives $2^{-4} \leqslant \delta$, that is $f(\Delta) \supset \Delta_{1 / 16}$.

LEMMA 3.2. Suppose that $f$ is a $K$-quasiregular harmonic mapping on the unit disc $\bar{\Delta}, f(0)=0$ and $|\operatorname{grad} f(0)| \geqslant 1$. Then for every $\theta \in \mathbb{R}$ there exists a point $w$ on the half-line $\Lambda_{\theta}$, which belongs to $f(\Delta)$, such that $|w| \geqslant c$, where $c=c(K)=2^{-6} / K$.

We call this result the Koebe lemma for quasiregular harmonic mappings with constant $c$.

PROOF. Let us first verify the decomposition property for quasiregular harmonic functions (shortly qrh): if $f$ is a $K$-quasiregular harmonic mapping, then $f=F \circ g$, where $F$ is an analytic function from $\Delta$ and $g$ is a $K$-quasiconformal mapping from $\Delta$ onto itself.

It is known that there is a quasiconformal mapping $g$ from $\Delta$ onto itself such that $g$ is a solution of Beltrami equation $g_{\bar{z}}=\mu g_{z}$ (see $\left.[\mathbf{A h 2}],[\mathbf{L V}]\right)$.

Let $F=f \circ g^{-1}$. Then we have for $\mu_{F}$ (see $\left.[\mathbf{A h 2}],[\mathbf{L V}]\right)$ that

$$
\mu_{F} \circ g=\frac{g_{z}}{\bar{g}_{\bar{z}}} \cdot \frac{\mu_{f}-\mu_{g}}{1-\mu_{f} \bar{\mu}_{g}}=0
$$

and we conclude that $F$ is a holomorphic function. Before we proceed with the proof, we give some remarks which are useful for understanding it.

Notes: Let us first observe that if $g$ is euclidean harmonic, then $L_{g}(0) \leqslant 2$. The following simple proof was suggested by the referee: Let $K_{r}$ be positively oriented circle of radius $r$ with the center at the origin. Then

$$
2 \pi i \partial g(0)=\int_{K_{r}} g(z) z^{-2} d z \text { for } r \in(0,1) .
$$

Hence by $g(\Delta)=\Delta$, we find $|\partial g(0)| \leqslant 1$ therefore $L_{g}(0) \leqslant 2|\partial g(0)| \leqslant 2$.

Note that $g$ is harmonic with respect to the metric $d \tilde{s}^{2}=\tilde{\rho}(\zeta)|d \zeta|^{2}$, where $\tilde{\rho}=\left|F^{\prime}\right|^{2}$. Although, $g$ is not in general harmonic with respect to the euclidean metric, it turns out that one can estimate the distortion of $g$ at 0 . Namely, if $g$ is conformal at 0 , since $\mu_{f}=\mu_{g}$, we can apply Lemma 3.1 and it yields $L_{g}(0) \leqslant 16$ (see Case 1 below).

We now continue the proof. Without loss of generality, one can suppose that $g(0)=0$, and let $d g(0)=p^{*} d z+q^{*} d \bar{z}$.

Case 1: $q^{*}=0$. Now it is convenient to use the notation $f^{*}$ instead of $g$ and to write $f=F \circ f^{*}$ and $f$ in the form $f=\bar{g}+h$, where $h$ and $g$ are holomorphic functions on $\bar{\Delta}$. Since $\mu_{f}=\frac{\overline{g^{\prime}}}{h^{\prime}}, \mu_{f}=\mu_{f^{*}}$ and $\mu_{f^{*}}(0)=0, f^{*} /\left|p^{*}\right|$ satisfies the conditions of Lemma3.1, and hence $f^{*}(\Delta)$ contains an open disk of radius $\frac{\left|p^{*}\right|}{16}$ and therefore $\frac{1}{16}\left|p^{*}\right| \leqslant 1$, that is $\left|p^{*}\right| \leqslant 16$. 
Hence, since $L_{f}(0)=\left|F^{\prime}(0)\right| L_{f^{*}}(0)$, and $L_{f^{*}}(0)=l_{f^{*}}(0)=\left|p^{*}\right|$, it follows that $L_{f}=\left|F^{\prime}(0)\right|\left|p^{*}\right| \leqslant 16\left|F^{\prime}(0)\right|$. By hypothesis, $1 \leqslant L_{f}(0)$ and therefore $1 \leqslant$ $16\left|F^{\prime}(0)\right|$, that is $\left|F^{\prime}(0)\right| \geqslant 2^{-4}$. Hence, it follows from Lemma 1.1 [Koebe lemma for holomorphic functions] that the theorem holds for $f$ with the constant $c_{0}=2^{-6}$.

Note that the function $f_{0}^{*}=L \circ f^{*}$ satisfies conditions of Lemma 3.1 in general, where $L=\left(A^{*}\right)^{-1}$ and $A^{*}(\zeta)=p^{*} \zeta+q^{*} \bar{\zeta}$; and, in particular, if $f^{*}$ is euclidian harmonic then $\left|p^{*}\right| \leqslant 1$.

Case 2: $q^{*} \neq 0$. Let $d f(0)=p d z+q d \bar{z}$ and $A=d f(0)$, that is $A(\zeta)=p \zeta+q \bar{\zeta}$; let $B=A^{-1}$ and $f_{0}=B \circ f$. Since $f_{0}$ is harmonic and $d f_{0}(0)=I d$, it satisfies the conditions of the previous case. Thus the theorem holds for $f_{0}$ with constant $c_{0}=2^{-6}$. By the hypothesis $L_{f}(0) \geqslant 1$, and therefore $l_{f}(0) \geqslant 1 / D(0)$, where $D(0)=L_{f}(0) / l_{f}(0)$ is the dilatation of $f$ at 0 . Hence, since $f=A \circ f_{0}$, and $A\left(\Delta_{c_{0}}\right)$ contains an open disk of radius $r_{0}=l_{f}(0) \cdot c_{0}=(|p|-|q|) \cdot c_{0}$, it follows that the theorem holds for $f$ with constant $l_{f}(0) 2^{-6}$ and therefore with constant $2^{-6} / D(0)$. Since, $K \geqslant D(0)$, it holds with constant $c(K)$.

As an application of the Koebe lemma for quasiregular harmonic mappings, we immediately obtain the following result, which we call the Koebe theorem for quasiregular harmonic mappings.

THEOREM 3.2 (Koebe theorem for quasiregular harmonic mappings). Suppose that $f$ is a $K$-quasiregular harmonic mapping on $\bar{B}$, where $B=B(a ; r)$; let $D=$ $f(B)$, and $f(a)=b$. Then for every $\theta \in \mathbb{R}$, there exists a point $w$ on the half-line $\Lambda_{b}^{\theta}$, which belongs to $f(\bar{B})$, such that $|w-b| \geqslant R_{f}(a)$, where $R_{f}(a):=c(K) r|\operatorname{gradf}(a)|$. In particular, there exists a point $\omega \in f(\bar{B})$ such that $|\omega|-|b|=|\omega-b| \geqslant R_{f}(a)$.

Now, it is clear that one can prove a version of Theorem A for quasiregular harmonic mappings by means of the Koebe theorem for quasiregular harmonic mappings, using a similar procedure as in the case of holomorphic functions. It is left to the interested reader as an exercise (see also [MM1]).

The following example shows the constant $1 / 16$ is sharp for quasiconformal mappings with dilatation $|\mu(z)| \leqslant|z|$.

ExAmple 4. Let $A(z)=4 z /(1+|z|)^{2}, k^{0}(z)=z(1-z)^{-2}$ and $f=k^{1}=k^{0} \circ A / 4$. By a straightforward calculation, one can verify that $k^{1}(\Delta)$ contains the disk of radius $1 / 16$, that $1 / 16$ is on the boundary of $k^{1}(\Delta)$ and that $\left|f_{\bar{z}}\right|=|z|\left|f_{z}\right|$.

Concerning the harmonic Koebe theorem, Clunie and Sheil-Small in [Cl-Sh] paper, mentioned that they first had found a proof with a weaker constant, and then Hinkkanen suggested to use the metric which gave $1 / 16$, the best constant which the method could produce.

There is conjecture that the best constant is $1 / 6$ in the harmonic analogy of the Koebe one-quarter theorem (see $[\mathbf{D u}]$ ).

\section{Appendix}

In this section, for convenience of the reader, we prove classical Schwarz lemma and derive some corollaries from it. Also a proof of the gradient growth lemma, 
which roughly states that: if gradient of a function satisfies certain type of growth, then that function belongs to Lipschitz-type spaces, is given.

Proposition 4.1 (Schwarz lemma). Let $B=B(a ; r)$ and $B^{*}=B(0, M)=$ $\Delta_{M}$ be two disks and $\varphi: B \rightarrow B^{*}$ be a holomorphic function. Then $r\left|\varphi^{\prime}(a)\right| \leqslant M$.

Apply the Cauchy inequality to $\varphi$ on $B$.

Proposition 4.2 (The subordination principle). Let $f$ and $F$ be holomorphic function on $\Delta$. If $f$ is univalent, $F(\Delta) \subset f(\Delta)$ and $F(0)=f(0)=b$, then $\left|F^{\prime}(0)\right| \leqslant\left|f^{\prime}(0)\right|$.

Proof. Apply Schwarz lemma to the function $\varphi=f^{-1} \circ F$ and use $\varphi^{\prime}(0)=$ $\left(f^{-1}\right)^{\prime}(b) F^{\prime}(0)$.

4.1. The estimate of modulus of derivative by means of the modulus of continuity. Let $B=B(a ; r), f$ holomorphic function on $B, f(a)=b$ and $M=:\{\sup |f(z)|: z \in B\}<+\infty$. If $M=1$, then $f: B \rightarrow \Delta$. Applying Schwarz lemma to the function $\varphi_{b} \circ f$, gives

$$
\left|f^{\prime}(a)\right| \leqslant \frac{1-|b|^{2}}{r},
$$

and hence $\rho=\rho_{f}(a)=: r\left|f^{\prime}(a)\right| / 2 \leqslant 1-|b|$. If $0<M<+\infty$, an application of this result to the function $f / M$, gives $\rho=: r\left|f^{\prime}(a)\right| / 2 \leqslant M-|b|$. Hence, if, in addition, $f$ is continuous on $\bar{B}$, then there exists $z_{1} \in \bar{B}$ such that

$$
\rho_{f}(a) \leqslant\left|f\left(z_{1}\right)\right|-|b| \text {. }
$$

Hence

$$
\rho_{f}(a) \leqslant \omega_{|f|}(r) .
$$

This gives the estimate of modulus of derivative by means of the modulus of continuity of $|f|$ and recall provides a key inequality for Dyakanov result.

We can rewrite the inequality (4.1)) in the form $R=\rho+|b| \leqslant\left|f\left(z_{1}\right)\right|$. Hence, there exists $z_{1} \in \bar{B}$ such that $f\left(z_{1}\right)$ does not belong to $\Delta_{R}$.

4.2. Estimate. The proof of the next lemma is based on the following:

EXERCISE 1. If $0 \leqslant a \leqslant b<1$, then

$$
I(a, b):=\int_{a}^{b}(1-x)^{\alpha-1} d x \leqslant \alpha^{-1}(b-a)^{\alpha} .
$$

Proof. By fundamental theorem of calculus, $I(a, b)=F(a)-F(b)$, where $F(x)=\alpha^{-1}(1-x)^{\alpha}$. Since $(1-a)^{\alpha} \leqslant(1-b)^{\alpha}+(b-a)^{\alpha}$, then $F(a)-F(b) \leqslant \alpha^{-1} \delta^{\alpha}$, and hence $I(a, b) \leqslant \alpha^{-1}(b-a)^{\alpha}$.

LEMmA 4.1 (Gradient growth lemma). If $u: \Delta \rightarrow \mathbb{C}$ and

$$
|\operatorname{grad} u(z)| \leqslant K(1-|z|)^{\alpha-1}, \quad z \in U
$$

for some $\alpha \in(0,1)$, then $u \in \operatorname{Lip} \alpha$ with the constant $M_{\alpha}=\left(1+2 \alpha^{-1}\right) K$. 
Proof. Let $a \in \Delta, b \in \Delta$ such that $0<|a| \leqslant|b|<1$. Suppose $\delta=|b-a|<1$ and set $a^{\prime}=\frac{1-\delta}{|a|} a, b^{\prime}=\frac{1-\delta}{|b|} b$. Note that $\left|a^{\prime}\right|=\left|b^{\prime}\right|=1-\delta$. We consider three cases according to the position of points $a$ and $b$ w.r. to the ring

$$
V=V_{\delta}=\{1-\delta<|z|<1\} ;
$$

and use the Exercise. In this way we indicate the geometry behind the proof.

(i) If $\delta \leqslant 1-|b|$, that is $b \notin V$, then $|\operatorname{grad} u| \leqslant K \delta^{\alpha-1}$ on the interval $[a, b]$. Hence, $|u(a)-u(b)| \leqslant K \delta^{\alpha}$.

(ii) If $1-|b|<\delta \leqslant 1-|a|$, that is $a \notin V$ and $b \in V$, then $\left|a-b^{\prime}\right| \leqslant|a-b|$ and

$$
|u(a)-u(b)| \leqslant\left|u(a)-u\left(b^{\prime}\right)\right|+\left|u\left(b^{\prime}\right)-u(b)\right| .
$$

The first term is estimated as in the case by (i). By the Exercise, the second term is estimated by

$$
K \int_{1-\delta}^{|b|}(1-x)^{\alpha-1} d x<\alpha^{-1} \delta^{\alpha}
$$

(iii) If $1-|a|<\delta$, that is $a \in V$ and $b \in V$, then

$$
|u(a)-u(b)| \leqslant\left|u(a)-u\left(a^{\prime}\right)\right|+\left|u\left(a^{\prime}\right)-u\left(b^{\prime}\right)\right|+\left|u\left(b^{\prime}\right)-u(b)\right| .
$$

The first and the third term are estimated by the Exercise as in the case (ii), the second as in the case (i).

\section{References}

[Ah2] L. Ahlfors, V., Lectures on Quasiconformal Mappings, Princeton, 1966.

[AMM] I. Anić, V. Marković, M. Mateljević, Uniformly Bounded Maximal $\Phi$-discs, Bers Spaces and Harmonic Maps, Proc. Amer. Math. Soc. Vol. 128 (10) (2000), 2947-2956.

[Cl-Sh] J. Clunie, T. Sheil-Small, Harmonic univalent function, Ann. Acad. Sci. Fenn. A I Math. 9 1984, 3-25.

[Du] P. L. Duren, Univalent functions, Springer-Verlag, 1983.

[Dyk1] K. M. Dyakonov, Equivalent norms on Lipschitz-type spaces of holomorphic functions, Advances in Math. 178 (1997), 143-167.

[Dyk2] K. M. Dyakonov, Holomorphic functions and quasiconformal mappings with smooth moduli, Adv Math. 187 (2004), 146-172.

[He-Sc] W. Hengartner, G. Schober, Univalent harmonic function, manuscript.

[He-Po] W. Hengartner, D. Poulin, Distortion theorems for diffeomorphisms, Canad. Math. Bull. 37:3 (1994), 351-360

[LV] O. Lehto, K. I. Virtanen, Quasiconformal Mappings in the Plane, Springer-Verlag, 1973.

[MM1] M. Mateljević, A version of Bloch theorem for quasiregular harmonic mappings, Rev. Roum. Math. Pures. Appl. 47:5-6 (2002), 705-707.

[MM2] M. Mateljević, Dirichlet's principle, distortion and related problems for harmonic mappings, Publ. Inst. Math., Nouv. Sér. 75(89) (2004), 147-171

[MM3] M. Mateljević, Quasiconformal and Quasiregular harmonic analogues of Koebe's Theorem and Applications, Ann. Acad. Sci. Fenn. 32 (2007), 301-315.

[P] M. Pavlović, On Dyakonov's paper "Equivalent norms on Lipschitz-type spaces of holomorphic functions", Acta Math. (Djursholm) 183 (1999), 141-143.

[P1] M. Pavlović, Lipschitz conditions on the modulus of a harmonic function, Rev. Mat. Iberoamericana 23:3 (2007), 831-845. 
[R] W. Rudin, Function Theory in the Unit Ball of $C^{n}$, Springer-Verlag, Berlin-HeidelbergNew York, 1980.

[Ri] S. Rickman, Quasiregular Maps, Springer-Verlag, Berlin-Heidelberg, 1993

Matematički fakultet

Beograd, Serbia

miodrag@matf.bg.ac . yu 